\title{
Um Relato de Experiência sobre o Uso do Pensamento Computacional para Potencializar o Ensino de Ciências na Rede Básica de Ensino
}

\author{
Thiago Moreira-Almeida ${ }^{1}$, Thais Castro ${ }^{2}$, Bruno Gadelha ${ }^{2}$ \\ ${ }^{1}$ Programa de Pós-Graduação em Educação em Ensino de Ciências e Matemática \\ (PPGECIM - Universidade Federal do Amazonas (UFAM) Caixa Postal 15.064 - \\ 91.501-970 - Amazonas - AM - Brazil \\ ${ }^{2}$ Instituto de Computação (ICOMP) - Universidade Federal do Amazonas (UFAM) \\ Caixa Postal 15.064 - 91.501-970 - Amazonas - AM - Brazil \\ tmda1706@gmail.com, thais@icomp.ufam.edu.br, bruno@icomp.ufam.edu.br
}

\begin{abstract}
Computational thinking is a skill which in order for students to master it is mandatory it become part of the subjects' school curricula, especially in STEM (Science, Technology, Engineering and Mathmatics) subjects. Fulfilling this idea, this work consists of planning and appying pedagogical activities with 6th grade students at the middle school level in Brazil, carried on a public school in Manaus city. The main purpose was to teach programming rudiments and, at the same time, reinforcing the study of ecology related to the curricular discipline of Natural Sciences. For achieving a most fast approach with immediate feedback we used Scratch programming language. The results indicate that there is a positive relation between the teaching of programming rudimentos and the increase of students's interest when traditional contente is associated with a technological teaching tool.
\end{abstract}

Resumo. O pensamento computacional é uma habilidade que para que os alunos adquiram é essencial que a mesma esteja aos currículos das disciplinas da escola, especialmente as correspondentes a temas de STEM (Ciência, Tecnologia, Engenharia e Matemática). Atendendo a essa ideia, este trabalho consiste no planejamento e aplicação de atividades pedagógicas com alunos do $6^{\circ}$ ano do Ensino Fundamental de uma escola pública da cidade de Manaus. $O$ principal objetivo foi o de ensinar rudimentos de programação e, ao mesmo tempo, reforçar o estudo de ecologia à disciplina curricular de Ciências Naturais. Para atingir uma abordagem mais rápida com feedback imediato usamos a linguagem de programação Scratch. Os resultados indicaram que existe uma relação positiva entre o ensino de rudimentos (lógica) de programação e o aumento do interesse dos alunos quando se associa um conteúdo tradicional a uma ferramenta tecnológica de ensino. 
VIII Congresso Brasileiro de Informática na Educação (CBIE 2019)

Anais do XXV Workshop de Informática na Escola (WIE 2019)

\section{Introdução}

A rede de educação básica brasileira possui como componente curricular o ensino de Ciências Naturais como disciplina que abarca o estudo da natureza. Esta disciplina passa por mudanças significativas onde são estabelecidas formas de aprendizado por competências e habilidades, além de propor o uso das tecnologias de comunicação e informação, para assim, criar habilidades interdisciplinares num contexto transdisciplinar. Estas mudanças são diretrizes estabelecidas através dos PCNs (Parâmetros Curriculares Nacionais).

A aplicação de software na educação é um recurso pedagógico que visa o desenvolvimento intelecto-cognitivo e pode ser utilizado para as aulas de diferentes disciplinas, incentivando a descoberta de informações e a construção do conhecimento. Esta ferramenta atua em um processo de ensino-aprendizagem mais dinâmico, que possui o interesse de não apenas ensinar informática para os alunos, mas também ensinar conteúdos interdisciplinares com a interatividade proporcionada pelo computador [Pimentel, 2011]. Um computador com acesso à internet é uma janela para conhecer o mundo sem sair da escola, essa janela permite que o aluno alcance o conhecimento e descubra novas fontes de aprendizagem. Dessa forma, a informática tem um potencial recurso pedagógico no ambiente escolar e por isso a escola precisa utilizar o computador e suas ferramentas como meio facilitador do processo de ensino-aprendizagem [Moraes, 1997]

Considerando os interesses e as exigências da sociedade atual e a necessidade de adequar o ensino às mudanças sociais, é preciso integrar a informática ao currículo escolar, pois os computadores são uma realidade do cotidiano, e a escola deve preparar o aluno para o mundo. No Brasil, a computação ainda é pouco explorada na Educação Básica e seus fundamentos, muitas vezes, acabam por ficar restrito apenas para aqueles que optam por cursos técnicos ou graduação na área [Valente, 2016]. Diversas iniciativas nesse sentido estão sendo tomadas em países desenvolvidos, como por exemplo o caso da Austrália que vem substituindo as aulas tradicionais de história e geografia por softwares educacionais que utilizam a lógica de programação como base de ensino dessas disciplinas [Bornelli, 2015]. Dessa forma, considerando a importância deste tema e a necessidade de trabalhos que envolvam a transdisciplinaridade dos conteúdos tradicionais do ensino básico de Ciências Naturais utilizando a lógica de programação, este trabalho teve como objetivo desenvolver atividades modulares que exercitam o pensamento lógico atrelado às atividades de produção e reflexão relativas ao conteúdo de ecologia com alunos do $6^{\circ}$ ano do Ensino Fundamental de uma escola pública de Manaus.

\section{Refencial Teórico}

\subsection{Pensamento Computacional no Ensino Fundamental}

Raciocinar logicamente para resolver problemas é uma habilidade desenvolvida pelos profissionais da área da computação. Como a programação é fundamental para operar os computadores, a tendência é que esses profissionais pensem nos problemas de forma analítica e desenvolvam soluções em forma de algoritmos [Easterbrook, 2014]. Essa forma estruturada de pensar foi denominada de Pensamento Computacional que pode representar a contribuição mais importante da ciência da computação para o mundo e deve ser ensinado aos estudantes nas mais diversas disciplinas pois é composto por um conjunto de técnicas baseadas em conceitos computacionais para resolver problemas nas diversas áreas, sendo uma habilidade fundamental que deve ser desenvolvida ainda no período da infância pois será aplicado ao longo da vida do ser humano [Wing 2006] 
VIII Congresso Brasileiro de Informática na Educação (CBIE 2019)

Anais do XXV Workshop de Informática na Escola (WIE 2019)

O ensino de Pensamento Computacional se mostra, além de motivador para inserção dos estudantes de ensino básico em Computação, uma ferramenta para o desenvolvimento de habilidades relacionadas à construção do pensamento de maneira lógica, tornando o ensino de programação mais acessível para um maior número de indivíduos e estimulando muitas capacidades cognitivas [Scaico et al. 2013].

\subsection{Computação Desplugada}

A Computação Desplugada consiste em atividades práticas que visam ensinar conceitos da computação por meio de atividades manuais e lúdicas, consiste em produção e realização de atividades sem a necessidade de utilizar programas de software ou hardware, de forma concreta, vivenciando a programação [Bell and Fellows 2007]. Diversos estudos têm mostrado a relevância da Computação Desplugada para a difusão das diferentes estratégias do Pensamento Computacional, por permitir que exista adequação para diferentes realidades escolares, visto que não requer o uso específico de um computador, bem como desmistifica a ideia de que o computador resolve problemas sem intervenção humana [Vieira and Barreto 2013]. Dentre as atividades possíveis de executar a Computação Desplugada, cita-se atividades como:

- "Caça ao Tesouro". Baseia-se em uma analogia aos programas de computador que precisam processar uma sequência de símbolos como letras ou palavras em um documento, ou até mesmo o texto de outro programa. Cientistas da computação freqüentemente usam autômatos de estados finitos para isso. Um Autômato de Estados Finitos segue um conjuto de instruções para verificar se o computador reconhecerá a palavra ou conjunto de símbolos.

- "Codificação e Decodificação". Utiliza o codigo binário para converter em textos e vice-versa. Inicialmente, deve-se decodificar uma mensagem de texto em código binário e posteriormente os alunos devem codificar outra mensagem com código binário em texto utilizando uma tabela de relações entre os dois códigos.

- "Decodificando a Amarelinha". Carecteriza-se como uma atividade que introduz o ensino da linguagem de programação de uma maneira fácil e divertida, menos abstrata e mais concreta, ao adaptar o jogo de amarelinha ao ensino de lógica de programação. Com papelão e giz podemos realizar uma atividade de programação desplugada para que as crianças criem códigos e símbolos com a finalidade de escrever um programa para jogar a amarelinha. A atividade incorpora o uso do corpo na aprendizagem de conceitos matemáticos, espaciais e de lógica de programação materializando-os no chão da sala de aula de uma forma lúdica e colaborativa.

- "Jogo da Velha Funcional". Funciona como o tradicional Jogo da Velha, porém adaptado para o uso do corpo como forma de interação. Constitui um importante recurso didático que é utilizado para trabalhar a atenção, a cooperação em grupo, além de estimular a capacidade de estratégia, de análise e de raciocínio lógico.

Os trabalhos científicos que envolvem o ensino de programação, Computação Desplugada e jogos digitais têm se destacado como uma das principais estratégias de aplicação da computação para o desenvolvimento do Pensamento Computacional em alunos do ensino regular básico [Bordini et al. 2016].

\subsection{Ferramentas ao Suporte Educacional}

Ambientes visuais de programação facilitam o desenvolvimento de software em um contexto que é divertido e não ameaçador [Meerbaum-Salant et al. 2013]. Dessa forma, o 
VIII Congresso Brasileiro de Informática na Educação (CBIE 2019)

Anais do XXV Workshop de Informática na Escola (WIE 2019)

desafio de favorecer o desenvolvimento de habilidades cognitivas, e levar o pensamento computacional aos alunos do ensino fundamental, passa pela necessidade de sugerir ferramentas que possam apoiar a aprendizagem de conceitos de lógica de programação de uma forma mais lúdica e adequada à faixa etária.

Alguns ambientes de programação foram testados e avaliados, mostraram-se não intuitivos, sendo os resultados não significativos [Kereki 2008]. Dentre estes ambientes, podemos citar os softwares: Alice, Jeroo e GreenFoot. Trabalhos científicos vêm demonstrando que o software Scratch se caracteriza como ferramenta com grande potencial a ser utilizada em projetos educacionais, pois tende a encorajar jovens a manter atitudes positivas sobre Ciência da Computação [Martins 2012, Scaico 2012, Von Wangenheim et al. 2014]. Esse ambiente traz uma linguagem que contribui para a aprendizagem de programação através de um conceito inovador de desenvolvimento de código orientado a projeto, que privilegia a computação criativa. Computação criativa é o termo utilizado para reconhecer que o conhecimento e as práticas que os jovens precisam adquirir para criar software devem ser provenientes dos seus interesses pessoais [Scaico 2013].

O software Scratch foi idealizado e desenvolvido por uma equipe de investigação do Media Laboratory do Massachusetts of Institute of Technology (MIT). O Scratch é um ambiente de programação visual que permite a criação, simulação de demonstrações, histórias animadas, games, tutoriais e outros programas interativos através de blocos programáveis que lembra o sistema Lego. Desde 2013 o Scratch está disponível na forma online ou por meio do download gratuito para diversos sistemas operacionais. Um dos principais objetivos do Scratch é introduzir noções de linguagem de programação como uma primeira experiência por se tratar de um software que não exige do seu usuário o conhecimento prévio sobre linguagem de programação. Além disso, ele pretende facilitar a introdução de conceitos de matemática, enquanto instiga o pensamento criativo, o raciocínio sistemático e o trabalho colaborativo.

Atualmente, estima-se que possa ser utilizado por todas as idades, pelo seu layout simples, com janela única e utilização mínima de comandos. Ele estimula a atenção da criança para o desenvolvimento das atividades de forma lógica. Nele, a programação dos blocos foi concebida para se encaixarem apenas de forma que façam sentido sintaticamente, além de permitir a manipulação através da exploração e compartilhamento de suas próprias produções. As construções no Scratch podem iniciar pela definição do palco (plano de fundo) e dos objetos. Os objetos são arrastados e soltos no local desejado, onde vão se encaixando, geralmente associados a imagens, sons e movimentos entre outras programações

Quando agrupados, surge uma programação, sem erros sintáticos [Malan and Leitner, 2007]. No Brasil, o professor tem a sua disposição no website conteúdos que vão de tutoriais, jogos, videoaulas, atividade e animações.

Com o objetivo de agregar o uso de uma ferramenta que possa ser utilizada em sala de aula e principalmente no ensino de ciências, esse projeto corrobora com os demais trabalhos que demonstram a importância dessa ferramenta como ambiente virtual educacional associado ao Pensamento Computacional.

\section{Métodos}

Segundo [Siva and Silva, 2016] o ensino de algoritmos pode ser introduzido por meio de ferramentas envolvendo plataformas virtuais como o Scratch ou por meio da computação desplugada. Inicialmente, o projeto foi orientado através da metodologia envolvendo a Computação Desplugada pois os recursos físicos, como computadores e tablets, eram bastante limitados. Outro ponto a ser mencionado diz respeito à necessidade de desmistificar a ideia entre os alunos de que o computador resolve os problemas sem a intervenção humana. 
VIII Congresso Brasileiro de Informática na Educação (CBIE 2019)

Anais do XXV Workshop de Informática na Escola (WIE 2019)

Dessa forma, os primeiros contatos com os alunos aconteceram através da realização de módulos de atividades com o objetivo de introduzir conceitos de computação tais como algoritmos, linguagem binária e programação. Os alunos participantes do projeto cursavam o $6^{\circ}$ ano de uma escola pública municipal. Os conteúdos apresentados eram acompanhados de exemplos práticos e simples do cotidiano como atravessar uma rua ou trocar as pilhas de um controle remoto. Essas simples atividades requerem um planejamento e ordenamento de passos para sua execução. Explicitar tal planejamento exige do aluno raciocínio lógico para resolução de problemas.

Em seguida, realizou-se uma oficina de Computação Desplugada envolvendo raciocínio lógico associado a movimentos do corpo para realização das atividades "Decodificando a Amarelinha" e "Jogo da Velha Funcional" descritas anteriormente. Foram, então, realizadas entrevistas visando a seleção de alunos para a etapa seguinte do projeto que visava utilizar os conceitos aprendidos na fase anterior em um ambiente computacional. No caso, o ambiente adotado foi o Scratch. A seleção dos alunos foi necessária uma vez que o laboratório da escola, chamado "Telecentro", dispunha de apenas 10 computadores para a atividade. A seleção teve como base suas notas nas disciplinas regulares de matemática e ciências naturais, Além da aptidão de cada aluno com relação à tecnologia. Foram selecionados um total de 20 alunos para o projeto.

As aulas do projeto ocorriam semanalmente no contraturno dos alunos, horário vespertino, pois a carga horária letiva no turno matutino estava totalmente completa por outras atividades pedagógicas. Aconteceram, no total, 9 aulas cujo objetivos estão descritos na Tabela 1 a seguir:

Tabela 1. Planejamento dos módulos do projeto.

\begin{tabular}{|c|l|}
\hline Módulo & \multicolumn{1}{|c|}{ Objetivo } \\
\hline $\mathbf{1}$ & $\begin{array}{l}\text { Apresentar aos alunos sobre os objetivos do projeto, características e } \\
\text { especificidades dos assuntos, importância da presença dos alunos em cada } \\
\text { encontro e qual seria o software educacional a ser trabalho pelos alunos. }\end{array}$ \\
\hline $\mathbf{2}$ & $\begin{array}{l}\text { Apresentar aos alunos os diferentes campos da computação e a apresentação } \\
\text { de escolas que já possuem a Ciência da Computação como disciplina regular } \\
\text { em seus currículo ao redor do mundo como, por exemplo, Estados Unidos da } \\
\text { América e Austrália. Apresentar aos alunos documentários e relatos de } \\
\text { crianças que já utilizam linguagens de programação para resolver problemas } \\
\text { cotidianos e desenvolver programas básicos de computadores. }\end{array}$ \\
\hline $\mathbf{3}$ & $\begin{array}{l}\text { Realizar a atividade computação desplugada denominada de "Caça ao } \\
\text { Tesouro", introduzindo os alunos aos conceitos de sequenciamento de } \\
\text { atividades que são essenciais à programação de computadores. }\end{array}$ \\
\hline $\mathbf{4}$ & $\begin{array}{l}\text { Realizar a atividade "Codificação e Decodificação" visando introduzir os } \\
\text { alunos aos conceitos de números binários. }\end{array}$ \\
\hline $\mathbf{5}$ & $\begin{array}{l}\text { Apresentar o software educacional Scratch e sua forma de construção de } \\
\text { algoritmos em blocos de programação lógicos. }\end{array}$ \\
\hline $\mathbf{6}$ & $\begin{array}{l}\text { Apresentar as especificidades e caracteristicas do software através de } \\
\text { exercícios e tutoriais disponibilizados pelo site http://scratchbrasil.net.br/. }\end{array}$ \\
\hline $\mathbf{7}$ & Realizar exercícios disponibilizados pelo site do http://scratchbrasil.net.br/. \\
\hline $\mathbf{8}$ & $\begin{array}{l}\text { Realizar a transdiciplinaridade entre o conteúdo de Ecologia em que se trabalha } \\
\text { cadeias alimentares e fluxos de energia na discplina curricular de Ciências } \\
\text { Naturais com o conteúdo de Ciência da Computação. }\end{array}$ \\
\hline
\end{tabular}


VIII Congresso Brasileiro de Informática na Educação (CBIE 2019)

Anais do XXV Workshop de Informática na Escola (WIE 2019)

9 Realizar entrevistas com o objetivo de observar o impacto do projeto no aprendizado do ensino de Ciências Naturais e aplicar um questionário para analisar o grau de satisfação dos alunos acerca dessa nova metodologia de ensino.

A seguir são apresentados os resultados da aplicação das atividades do projeto com os alunos. Tais resultados estão baseados nas observações dos professores, entrevistas e questionários com os alunos participantes e análise do desempenho destes alunos na disciplina Ciências Natuais.

\section{Resultados e Discussão}

O presente projeto destacou atividades com o objetivo de desenvolver o pensamento lógico e computacional dos alunos visando solucionar diferentes problemas de maneira racional e intuitiva através de blocos de programação no software educacional. Com a aprendizagem das principais estruturas de programação utilizando as ferramentas do software, os alunos puderam desenvolver o assunto de ecologia através do tema de cadeias alimentares, dentro do componente curricular de Ciências Naturais.

Durante o desenvolvimento do tema, os alunos foram reunidos em grupos de 05 integrantes. Cada grupo recebeu uma folha com instruções explicando a atividade e um computador para elaborar o algoritmo solicitado. Então, os alunos escolheram fotos de animais na Internet e montaram uma cadeia alimentar com as seguintes caracteristicas: 04 níveis tróficos, 01 produtor, 03 consumidores, pelo menos 01 réptil e pelo menos 01 inseto. Os personages deveriam locomover-se em fundo de tela característico de savana. No momento do encontro de um predador com uma presa, a presa deveria desaparecer. A ordem dos níveis tróficos deveria ser obedecida, ou seja, o $2^{\circ}$ nível trófico só poderia desaperecer quando o $1^{\circ}$ nível trófico desaparecesse e assim por diante.

Os alunos se sentiram confortáveis durante as atividades e desenvolveram soluções criativas que se concretizaram em diferentes versões de cadeias alimentares utilizando diferentes seres vivos que estão classificados dentro os cinco reinos biológicos. Assim, foi possível observar que os alunos relacionaram o conteúdo das aulas teóricas de ciências com as diferentes ações, comandos, cenários e demonstraram o total domínio em utilizar a ferramenta Scratch. Como objetivo principal, os alunos puderam visualizar os principais conceitos envolvidos na criação de um programa do software, desenvolvendo o raciocínio sequencial e a habilidade para resolver um dado problema utilizando técnicas inerentes à programação.

Em estudo realizado em [Meerbaum-Salant et al. 2013], os resultados mostraram que a maioria dos alunos é capaz de compreender os conceitos de Ciência da Computação. Em nosso caso, os resultados se mostraram bastante semelhantes. Os alunos se sentiram confortáveis com a temática abordada nos slides e as dúvidas que foram levantadas durante a explicação teórica puderam ser resolvidas de maneira eficiente pelos discentes, mostrando o tópico de programação de maneira visual no Scratch. Eventuais erros cometidos pelos alunos na construção de ações no Scratch foram facilmente contornados. Muitas das vezes sem intervenção do professor, pois os alunos conseguiram identificar seus próprios erros a partir do pensamento lógico.

Outro ponto a ser discutido é em relação à motivação dos alunos que se concretizou em um real engajamento durante a construção dos códigos relativos aos desafios de programação propostos. $O$ fato de não existir a preocupação com a sintaxe de uma linguagem de programação tradicional, e que a ferramenta apresenta os conceitos de programação sob a forma de quebra-cabeças, possibilitou que não houvesse desmotivação 
VIII Congresso Brasileiro de Informática na Educação (CBIE 2019)

Anais do XXV Workshop de Informática na Escola (WIE 2019)

por parte dos alunos, não havendo reclamação destes durante a execução das atividades, o que torna a execução desta atividade como bastante satisfatória.

Observou-se que a assimilação do conhecimento proposto pelo conteúdo programático foi atingida, pois todos os alunos foram capazes de construir as cadeias alimentares com todas as suas particularidades corroborando para o uso do Scratch como ferramenta educacional para a iniciação à programação. Na Figura 1, é apresentado um exemplo de animação desenvolvida em blocos no software Scratch por alunos do projeto. $\mathrm{Na}$ animação em questão a cadeia é iniciada com a fruta fornecida pela planta que é ingerida pelo inseto. O inseto é presa do sapo que, por sua vez, é presa da cobra.

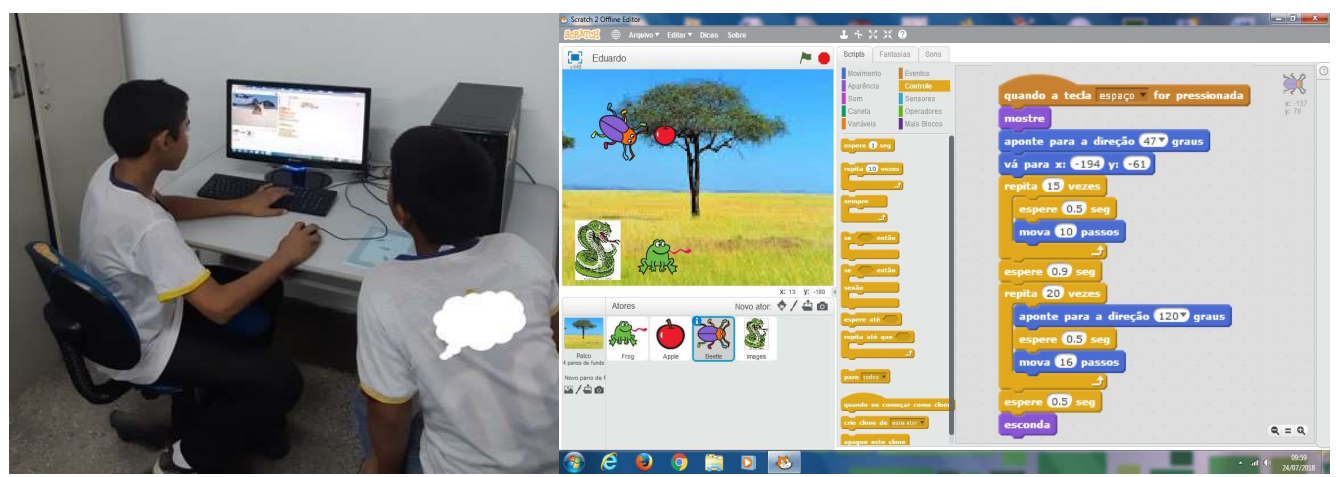

Figura 1. Alunos desenvolvendo animação sobre cadeia alimentar com Scratch.

Segunda a aluna A1, afirma que "As aulas de Ciências se tornaram mais legais porque agora eu consigo criar animais e decidir como acontece o movimento de um animal se alimentar do outro, como se fosse um jogo de vídeo game". Neste relato, é possível identificar que a aluna consegue relacionar o conteúdo de Ciências com a nova ferramenta tecnológica, além de ser a protagonista do conhecimento formado e utilizar os seres vivos que a rodeiam como parte formadora do conhecimento adquirido.

Outro relato é observado pelo aluno A2, "As atividades no Scratch são importantes porque agora eu uso a sala do telecentro (espaço reservado para a sala de mídias) e entendo porque nós devemos aprender como os computadores funcionam e como se faz um programa de computador". Observa-se que o discurso do aluno apresenta um grau de satisfação de conseguir relacionar a importância da computação com o aprendizado fornecido na escola e que esses dois universos podem caminhar paralelamente ao longo da vida escolar do educando.

Em relação aos questionários e entrevistas foi observado que $80 \%$ dos alunos obtiveram nota melhor que o bimestre anterior referente à disciplina curricular de Ciências Naturais. Apenas um dos alunos (A18) apresentou desempenho inferior ao do bimestre anterior ao do projeto. Vale destacar que este aluno desistiu de participar das atividades do projeto. Os alunos A3, A15 e A20, apesar de apresentarem desempenho superior ao bimestre anterior, esse aumento foi discreto. Esses alunos também desistiram no meio das atividades propostas. As notas detalhadas de cada aluno participante em cada bimestre, assim como o percentual da diferença entre elas, estão representadas na Tabela 2. As atividades foram desenvolvidas após o primeiro bimestre. Os alunos marcados com “*” desistiram das atividades. Também foi possível observar que $100 \%$ dos alunos da pesquisa responderam que gostaram da metodologia proposta e o mesmo percentual afirma que gostaria que a metodologia de ensino fosse aplicada aos demais conteúdos da mesma disciplina. 
VIII Congresso Brasileiro de Informática na Educação (CBIE 2019)

Anais do XXV Workshop de Informática na Escola (WIE 2019)

Tabela 2. Comparação das notas dos alunos, referentes às duas turmas envolvidas, antes e depois da aplicação da metodologia.

\begin{tabular}{|cccc|cccc|}
\hline & \multicolumn{3}{|c|}{ Turma 1 } & \multicolumn{5}{|c|}{ Turma 2 } \\
\hline Aluno & $\mathbf{1 ~ B i m ~}$ & 2 Bim & Diff(\%) & Aluno & 1 Bim & 2 Bim & Diff(\%) \\
\hline $\mathbf{A 1}$ & 8.2 & 9.1 & 9 & $\mathrm{~A} 11$ & 9.0 & 10,0 & 10 \\
\hline $\mathbf{A 2}$ & 7.5 & 9.0 & 15 & $\mathrm{~A} 12$ & 7.6 & 9.2 & 16 \\
\hline $\mathbf{A 3} *$ & 8.1 & 8.6 & 5 & $\mathrm{~A} 13$ & 8.2 & 9.1 & 9 \\
\hline $\mathbf{A 4}$ & 7.0 & 8.8 & 8 & $\mathrm{~A} 14$ & 8.8 & 9.8 & 10 \\
\hline $\mathbf{A 5}$ & 6.8 & 8.3 & 15 & $\mathrm{~A} 15^{*}$ & 7.6 & 7.9 & 3 \\
\hline $\mathbf{A 6}$ & 8.3 & 9.2 & 9 & $\mathrm{~A} 16$ & 7.8 & 9.6 & 18 \\
\hline $\mathbf{A 7}$ & 7.8 & 8.8 & 10 & $\mathrm{~A} 17$ & 8.9 & 9.8 & 9 \\
\hline $\mathbf{A 8}$ & 6.1 & 8.4 & 23 & $\mathrm{~A} 18^{*}$ & 8.8 & 8.6 & -2 \\
\hline $\mathbf{A 9}$ & 7.2 & 8.9 & 17 & $\mathrm{~A} 19$ & 8.3 & 9.2 & 9 \\
\hline $\mathbf{A 1 0}$ & 8.6 & 9.6 & 10 & $\mathrm{~A} 20^{*}$ & 7.5 & 8.0 & 5 \\
\hline
\end{tabular}

Por fim, o professor responsável pela disciplina de Ciências Naturais foi consultado acerca de suas impressões sobre a adequação da metodologia à sua disciplina. O professor afirmou: "Os meus alunos que foram submetidos a essa tecnologia educacional apresentaram um melhor aproveitamento na disciplina e apresentaram uma maior participação durante as aulas teóricas de ecologia da natureza, além de melhorarem o comportamento em relação a atenção e conversas paralelas.". Em sua fala, o professor destaca a melhoria da participação, comportamento e desempenho dos alunos nas aulas, dando indícios de que a abordagem utilizada é adequada para ser utilizada em sua disciplina.

\section{Conclusões}

Este artigo relatou uma experiência envolvendo o uso de pensamento computacional aplicado ao ensino de Ciências Naturais, mais especificamente no tópico de cadeias alimentares, na área de ecologia. A experiência envolveu o uso de uma abordagem que combinou atividades de computação desplugada com uso de ferramenta de programação em blocos, o Scratch. Os alunos sentiram-se motivados e engajados. Tal fato é sustentado pelo aumento do desempenho demonstrado pelos alunos no bimestre subsequente à aplicação da metodologia proposta.

Assim, foram desenvolvidos o raciocínio sequencial e a habilidade para resolver problemas utilizando técnicas inerentes à programação de computadores. Os resultados mostraram que a metodologia proposta cativou a atenção de crianças e os conceitos foram assimilados facilmente. Com isso, concluímos que a aprendizagem de Pensamento Computacional e Programação pode ser agradável e de fácil entendimento dos aprendizes.

Este trabalho de aprendizagem em Pensamento Computacional, aplicado em uma escola municipal de Manaus, teve como diferencial a transdiciplinaridade dos conceitos de ecologia como item na grade curricular da disciplina de Ciências Naturais através do desenvolvimento de atividades de construção de algoritmos que determinavam cadeias alimentares pelos alunos. Como contribuição desta experiência, tem-se uma abordagem sistemática, materializada no plano de atividade relatada, envolvendo tanto computação desplugada e plugada (com o uso do Scratch) a ser desenvolvida no ensino de Ciências. Os próximos passos consistem em generalizar a metodologia proposta para verificar sua adequação a outras disciplinas do currículo escolar do ensino fundamental. 
VIII Congresso Brasileiro de Informática na Educação (CBIE 2019)

Anais do XXV Workshop de Informática na Escola (WIE 2019)

\section{Agradecimentos}

Este projeto foi parcialmente financiado pelo Programa de Apoio a Jovens Doutores PJD da UFAM, Edital 041/2016 - PROPESP/UFAM e obteve suporte financeiro, para a participação deste evento, do Programa de Pós-Graduação em Ensino de Ciências e Matemática (PPGECIM), da UFAM.

\section{Referências}

Borneli, J. Austrália começa substituir disciplinas por Programação nas escolas, 2015. Disponível em: https://conteudo.startse.com.br/mundo/juniorboneli/australiacomecasubstituir-disciplinas-de-historia-e-geografia-por-aulas-de-programacao/. Acessado em: 28 de março de 2017.

Bell, T.; Witten, I. e Fellows, M. (2011). "Computer Science Unplugged - Ensinando Ciência da Computação sem o uso do Computador". Tradução de Luciano Porto Barreto, 2011. Disponível em: http://csunplugged.org/. Acesso em 02/02/2018.

Easterbrook, S. "From Computational Thinking to Systems Thinking: A conceptual toolkit for sustainability computing". Proceedings of the 2nd International Conference on Information and Communication Technologies for 70 Anais do XXI Workshop de Informática na Escola (WIE 2015) CBIE-LACLO 2015 Sustainability (ICT4S'2014), Stockholm, Sweden, 24-27 August, 2014.

De Kereki, I. F. (2008). Scratch: Applications in computer science 1. Frontiers in Education Conference. FIE 2008. 38th Annual. IEEE.

Malan, D. J. \& Leitner, H. H. (2007) Scratch for budding computer scientists. Proceedings do 38th SIGCSE'07, Kentucky, USA, 2007.

Martins, A. R. D. Q. (2012). Usando o Scratch para potencializar o pensamento criativo em crianças do ensino fundamental. Dissertação de Mestrado. UPF - RS.

Meerbaum-Salant, Orni, Michal Armoni, and Mordechai Ben-Ari (2013). Learning computer science concepts with Scratch. Computer Science Education 23.3: 239264.

Moraes, M. C. Informática Educativa No Brasil: Uma história vivida, algumas lições aprendidas. Revista Brasileira de Informática na Educação - Número 1 - 1997

PimentelL, M; Fuks, H (Org). Sistemas Colaborativos. Rio de Janeiro: Elsevier, 2011

Richtel, M. Programação vira disciplina em escolas infantis nos EUA, 2014. Disponível em: http://www1.folha.uol.com.br/tec/2014/05/1456608- programacao-vira disciplina- em- escolas- infantis-nos-eua.shtml.Acessado em: 20 de março de 2017.

Scaico, P. D., et al. (2013). Ensino de programação no ensino médio: Uma abordagem orientada ao design com a linguagem Scratch. RIBIE 21.02: 92.

Scaico, P. D., de Lima, A. A., da Silva, J. B. B., Azevedo, S., Paiva, L. F., Raposo, E. H. S., ... \& Mendes, J. P. (2012). Programação no ensino médio: uma abordagem de ensino orientado ao design com Scratch. In Anais do Workshop de Informática na Escola (Vol. 1, No. 1).

Silva, G.T. Da, Souza, J.L. De e Silva, L.A.M. Da (2016). Ferramenta Scratch para o Aprendizado de Programação no Ensino Fundamental I', in Anais dos Workshops do V Congresso Brasileiro de Informática na Educação (CBIE).

Valente, J. A. Integração do pensamento computacional no currículo da Educação Básica: diferentes estratégias usa das e questões de formação de professores e avaliação do aluno. Revista e Curriculum, São Paulo, v.14, n.03, p. 864 - 897, 2016. 
VIII Congresso Brasileiro de Informática na Educação (CBIE 2019)

Anais do XXV Workshop de Informática na Escola (WIE 2019)

Vieira, A. Passos, O. and Barreto, R. (2013). Um Relato de Experiência do Uso da Técnica Computação Desplugada, in Anais de Workshop sobre Educação em Computação(WEI).

Von Wangenheim, C. G., Nunes, V. R., \& dos Santos, G. D. (2014). Ensino de computaçao com scratch no ensino fundamental-um estudo de caso. Revista Brasileira de Informática na Educação, 22(3), 115-125.

Wing, J. M. (2006). Computational thinking. Communications of the ACM, 49(3), 3335. 